\title{
INTERTEXTUALIDAD Y SIMBOLISMOS: SU IMPORTANCIA EN LA TRADUCCION Y RECREACION DEL TEXTO LITERARIO
}

\author{
Ana María Dianda Martínez \\ Universidad Nacional, Costa Rica
}

\begin{abstract}
Este artículo forma parte del análisis de la macroestructura de la novela The Haunting of Hill House ${ }^{1}$. Destaca la necesidad de investigar las relaciones intertextuales y los simbolismos para la traducción literaria aportando numerosos ejemplos. El trabajo de graduación al que pertenece, La casa poseída ${ }^{2}$, traduce parcialmente la obra y desarrolla un método de traducción consistente en un proceso de investigación sucesiva y acumulativa de aspectos vinculados a la historia de la cultura y de la literatura así como a la teoría literaria, los que se confrontan con el texto y sus aspectos lingüísticos; se facilita así la interpretación y recreación de la novela en español.
\end{abstract}

Refiriéndose a Macbeth y hablando de la complejidad del mensaji. que encierra, como gran obra de arte, dice el escritor costarricense J()aquín Gutiérrez, en el prólogo de su traducción: “... hay además un nicicleo que escapa a todo intento de explicación racional,..."3. Salvandu las distancias, creo que la advertencia es válida para la comprensión

I Shirley Jackson, The Haunting of Hill House (Nueva York: Penguin Books, 1984). En adelante se hará referencia solamente al número de página.

Ana María Dianda Martínez, traductora, La casa poseída de Shirley Jackson, (Universidad Nacional, biblioteca Joaquín García Monge, Heredia, tesis 3104, 1999).

1 Joaquín Gutiérrez, traductor, "Prólogo" en William Shakespeare, Macbeth (San José: Editorial de la Universidad de Costa Rica, 1991) 14. 
cabal de cualquier texto literario. Siempre va a haber algo que no logramos aprehender y que muchas veces nos hace volver una y otra vez sobre sus páginas, porque nos atrae y nos dice más de lo que racionalmente podemos captar. El análisis de un texto literario, en este caso la novela The Haunting of Hill House, de la escritora estadounidense Shirley Jackson, con miras a su posterior traducción, entraña una tarea más exigente y minuciosa que la que reclama un texto que no lo es. Las razones surgen de las mismas complejidades de lo literario.

A pesar de sus dificultades, la traducción literaria, tarea fundamental para el desarrollo de la literatura y por ende de las culturas de los pueblos y del conocimiento del ser humano, ha sido abordada de manera ejemplar - muchas veces - en Latinoamérica y el resto del mundo. Los artífices de esas traducciones-recreaciones reconocidas y prestigiosas han marcado las pautas: unas veces elaborando teorías, otras a través de prólogos o comentarios críticos, enriqueciendo así la lectura del texto traducido, por medio del análisis de la obra y de su relación con el contexto cultural en que se produjo, tanto en un sentido espacial como diacrónico.

En el caso de la presente obra, el traductor se enfrenta con un reto especial: un marcado énfasis en el uso, deconstrucción y recreación de otros textos; descubrirlos y averiguar la función que desempeñan en la novela va a ser una de sus principales dificultades pero, al mismo tiempo, una de sus mayores gratificaciones. La autora busca la complicidad de sus lectores de una manera particular. A través de la rica incorporación de otros textos, de forma explícita en unos casos, aludidos o implícitos én otros, y más allá del efectivo manejo de las técnicas literarias, de la frescura en los diálogos, de las vívidas descripciones, de la prosa esmerada y el lenguaje poético, ofrece un caleidoscopio de mensajes entretejidos cuyo descubrimiento proporciona al lector satisfacciones adicionales, algo así como tener acceso a un texto lleno de múltiples códigos que permiten muchas lecturas complementárias. La presencia de este "discurso ajeno"-_lo que otros 
dijeron-, responde a una intención de la autora, palpable luego de la investigación de otros factores relacionados con la producción de la novela (la autora y su obra, autores que presumiblemente la influenciaron, el momento y el medio socio-cultural en que la novela surge en la cultura de origen) y su confrontación con el mismo texto. El traductor debe ser ese lector atento y dispuesto a entrar en el juego, capaz de descubrir esas conexiones, el "tono" y las circunstancias en que las intertextualidades se presentan en el texto, pero que se cuida de no decir más de lo que la autora deseaba, pues hay una característica inherente al género de la novela, la ambigüedad, que fascina al lector y que el traductor debe preservar.

\section{Intertextualidades}

Hablar de intertextualidad es referirse a cómo se conjugan las lecturas anteriores que ha hecho la autora y que de diversas formas incluye en su texto, y aquellas que ese mismo texto evoca en la mente de quien lo lee. Para Jonathan Culler, la obra literaria puede ser vista como una “...construcción intertextual —un producto de varios discursos culturales en los que se difunde para hacerse inteligible__..."4 Roland Barthes alude a esta intertextualidad del texto literario cuando afirma: "[el texto es] ...un espacio multidimensional en el que varias escrituras, ninguna de ellas original, se mezclan, chocan. El texto es un tejido de citas...", y luego agrega "El lector es el espacio en el que se inscriben todas las citas que conforman una estructura..." ${ }^{\text {. La novela }}$ de Jackson es rica en intertextualidades que surgen y se entretejen enriqueciendo sus significados.

En ella se cuentan las experiencias de un grupo de personas involucradas en la investigación de fenómenos paranormales en una

4. Jonathan Culler, Sobre la deconstrucción. Teoría y crítica después del estructuralismo (1982, ed. en español: Madrid: Cátedra, 1992) 34.

5. Roland Barthes, Image, Music, Text. n.d.: Hill Wang, 1977, 146-148, citado por Culler, 34. 
mansión llamada Hill House, supuestamente embrujada. El relato se centra en el personaje de Eleanor Vance, mujer solitaria y marcada por profundas heridas psicológicas, para quien el experimento se convierte en un verdadero viaje al infierno del inconsciente, viaje que se inicia como búsqueda de la salvación a través del amor y concluye en la destrucción del yo, la locura y la mucrtc.

El texto pertenece al género Gótic(), litcralura yuc explora los lados más oscuros de la mente por medio de imíngeness terroríficas que actúan como metáforas de miedos, ansicdades, (lescess prohibidos y secretos profundos que permanecen blopucarlos a la conciencia.

En The Haunting of Hill Housc hay mulliples lex los cuya presencia, explícita o insinuada, está íntimamconlc associal(al al sentido total de la obra y ofrece al lector un abanico de mensiljes y lcmiss. El mito de Eros y Psique, que a su vez subyace en linl(os cllcinlus de hadas, está presente como hilo conductor de la hisf(orial. Jiackson retoma esta leyenda: Psique encuentra al Amor en la graill ('issil (le la colina pero no puede verlo a la luz del día, ni sus hermalniss puc(len siaber quién es; teme que sea un monstruo, pero descubre (juc no lo es. liros al verse descubierto huye. Venus, celosa, somclc a P'sicjuc a las más tediosas tareas, que ella ejecuta pacientemente piral recup)(rillo y lo logra casándose con él con la complacencia de los diosis's. Pcru Jiackson relee la leyenda desde una óptica feminista: Illcanorr, la protagonista, viaja (viaje exterior e interior, dentro de sus micdos y sul inconsciente) en búsqueda del amor y lo encontrará en Ilill Ilousce, lil antigua casa victoriana de la colina que perteneció a llugh ( 'rilin y allornada con figuras de Cupido, que es la casa de Eros. (rec (juc conconlrará al Amor en el doctor Montague, científico maduro que enciarlia a unla autoridad paterna; lo busca en Luke, arquetipo clásico del scelucl(or (le mujeres; y cree hallarlo en Theodora, imagen femenina desenvuclla, independiente y sexualmente ambigua, pero las fucrtes expericncias psíquicas que enfrentará en Hill House, la llevan a un (cscloblamiento de su personalidad: por un lado, traumáticas vivencias incestucsals aparecen en sus pesadillas nocturnas; por el otro una figura invisible la abraza, 
le canta y la conforta. Encuentra al amor en el fantasma de Hugh Crain, que es sin duda otra figura paterna violenta y represiva(¿la suya?). Sin embargo, no podrá permanecer en esa casa, ni revelar su secreto y la parte de ella que no lo tolera la conduce al suicidio. La imagen de Venus es asumida con características negativas por la madre de Eleanor, personaje que reitera el mensaje de dominación patriarcal presente no sólo en las estructuras sociales sino en las literarias, como lo indican, desde una perspectiva femenina, Sandra M. Gilbert y Susan Gubar cuando afirman “.. the use of male devised plots, genres and conventions may involve a female writer in uncomfortable contradictions and tensions"6.

Este mensaje patriarcal está grabado en la mente de Eleanor y así el cuento de hadas aparece recurrentemente en su monólogo interior, no sólo su contenido, sino también formalmente. En el viaje hacia Hill House que se describe en el primer capítulo aparecen sucesivamente el buen leñador que da abrigo a la niña en el bosque cuando cae la noche -Blanca Nieves-, Cenicienta que limpia con esmero la casona y los leones y finalmente La bella durmiente, recreado con escenarios y personajes en el capítulo primero del texto original (1920) y en la versión traducida al español ${ }^{7}$. En el capítulo segundo se hace alusión a Barba azul: mentalmente, Eleanor llama en su ayuda a "sister Anne" (38), igual que lo hace la protagonista del cuento, y más adelante, en el capítulo 3 (que no forma parte de la traducción), jugando Eleanor dice, sin querer, que ama a alguien que tiene barba, de quien comentan que vive en Bangkok y que se ocupa de "molestar a las mujeres"(61). En el mismo capítulo se alude reiteradamente al cuento de Andersen, Las zapatillas rojas. La metáfora que surge de este cuento tradicional: el deseo irrefrenable por la danza --danzar hasta morir - simboliza una pasión vital y por extensión el arte femenino de acuerdo a Gilbert y Gubar ${ }^{8}$. Y este amor a una actividad

6. Sandra Gilbert y Susan Gubar, The Mad Woman in the Attic. The Woman Writer and the $19^{\text {th }}$ Century Literary Imagination (New Haven: Yale UP, 1984) 70.

7. Dianda Martínez, 21-23.

8. Gilbert y Gubar, 57. 
artística representa un sacrificio y una dedicación constante. En The Haunting of HillHouse Eleanor picnsa "con profunda satisfacción que sus pies son hermosos en sus sandalias rojas" ( red sandals), se define "desde los rojos dedos de sus pics (red locs) hasta la cabeza", "tener zapatos rojos (red shoes) va con scr lilcimor" y al rito agrega que tiene

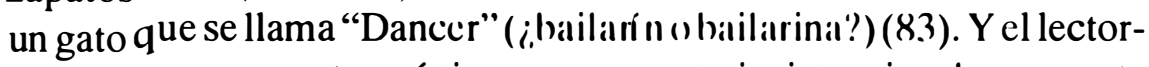
traductor se pregunta qué ticne (juc ver essla insistencia y la respuesta se aclara sólo por la metáfora del cucenlo. P’or un lialo la protagonista se atreve a hacer lo que sicmpre (fuisol y nunl'al sce animo, asumir su femineidad-sexualidad de una mallcera mlís a agresesival y lambién está la premonición: quien sucumbil a li lenlakcioul (le usiar lis zalpatillas rojas,

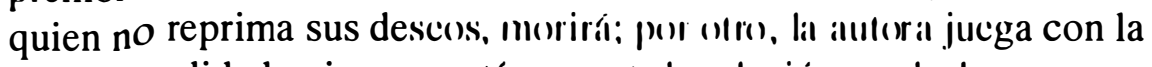

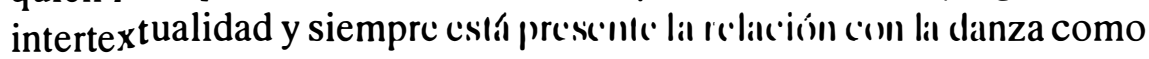
vocación artística. Por esos al Iradıc ir liss silludialiass sicmpre tendrán

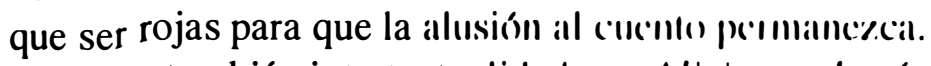

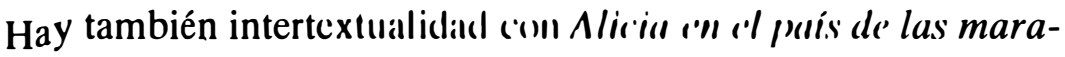

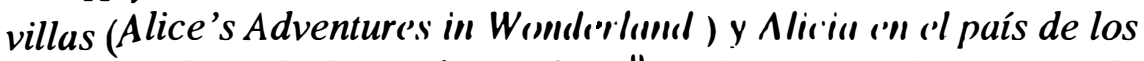
espejos (Through the Looking-(ilassis)". I il inlcrlexlllilidad se manifiesta, por ejemplo, en relación c(on) (') priml('r), ('n lis associación mental que hace Eleanor entre la somrisia hurlomil de M1. I )u(lley, cl cuidador

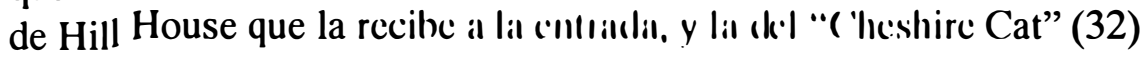

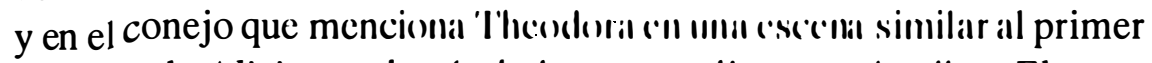
capítulo de Alicia en el pals de lus mururillas cuandlo clla y Eleanor

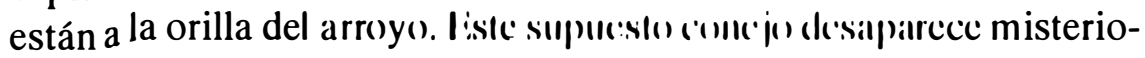
samente (54). Recordemos (juc: Aliciul ('slish)il ('olll sull licrmana en el

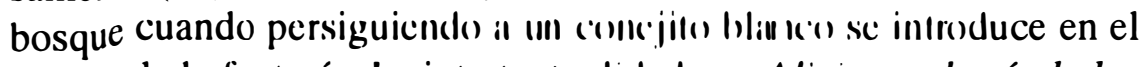

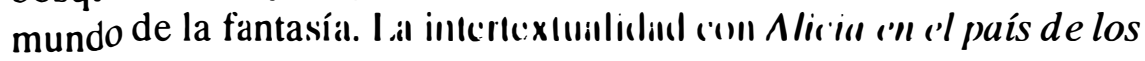

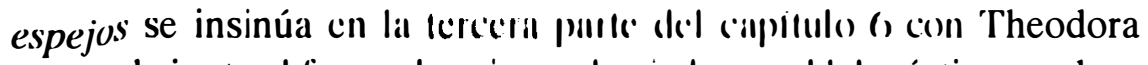

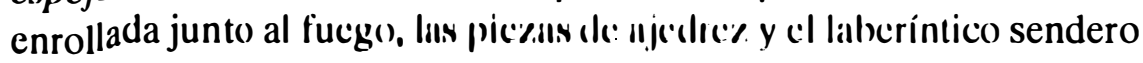

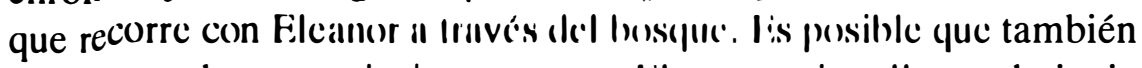
los huevos duros cue insistenlentenlle lilcamer (juicere llevar al picnic

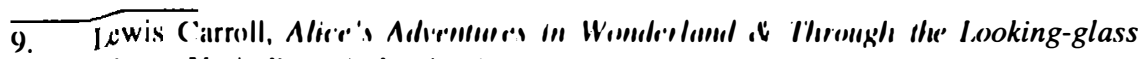
(Nucval York: I'conpuinu lluwikn, I')(nll) 
estén asociados con el famoso Humpty Dumpty de Alicia en el país d' los espejos y su petulante uso de las palabras. Recordemos que las dos "aventuras" de Alicia, cuyo tema central tienen que ver con el proceso de crecimiento de niña a mujer, forman parte de las obras maestras de la literatura victoriana y fueron escritas para entretener a tres niñas. Según Horace Gregory en el prólogo a la edición de ambos cuentos de Signet Classic 1960, Lewis Carroll (en realidad el reverendo Charles Lutwidge Dodgson), parodió a través de ellas la literatura infantil utilizada para educar a las niñas y futuras esposas del siglo XIX. Sin represiones, dentro de los “sueños" de Alicia, Dodgson se burla en esta “...alert and critical psychological novel” de la sociedad y ambigüedades de su época ${ }^{10}$.

La canción de amor que acude mutilada y con insistencia a la memoria de Eleanor es una antigua melodía tradicional, a la que Shakespeare puso letra, incorporándola a su comedia Noche de Reyes (Twelfth Night or What you Will), según lo indica Manuel Angel Conejero ${ }^{11}$. Ezequiel Martínez Estrada en su prólogo a las Comedias afirma que esta corrección ya figuraba en el Consort Lessons (1599) y en el Book of Ayres (1601) ${ }^{12}$. La canta Feste, un bufón, burlándose del atildado y enamoradizo Sir Andrew pretendiente de Olivia. La comedia transcurre en un lugar de fantasía, Iliria, y narra desencuentros amorosos y confusiones de género, aún mayores en la época de Shakespeare pues los personajes de las jóvenes doncellas debían ser interpretados por muchachos. El conflicto central surge cuando Viola, la protagonista, se disfraza de hombre y logra sin quererlo enamorar con apasionados versos a Olivia. Algunos críticos ven como tema

10. Horace Gregory, "Prólogo" en Lewis Carrol (Alice's Adventures in Wonderland \& Through the Looking-glass) vi y v-x.

11. Manuel Angel Conejero, "Prólogo" en William Shakespeare, Noche de Reyes o Como queráis - Twelfth Night or What you Will (edición bilingüe del Instituto Shakespearc dirigida por M.A. Conejero, Barcelona: Ediciones Altaya, 1994, 184).

12. Ezequiel Martínez Estrada, "Prólogo" en William Shakespeare, Noche de Reyes.s, Comedias (traducción Jaime Clark. Estudio preliminar de Ezequiel Martínez Estralia, Buenos Aires: W.M. Jackson, 1960) XXXIV. 
central la locura; tal es el caso de Barbara Melchiori en la introducción a la edición citada del Instituto Shakespeare ${ }^{13}$, mientras Martínez Estrada menciona como verdadero asunto el Amor ${ }^{14}$. La clave de la relación entre ambos textos bien podría estar en el dúo Viola-Olivia, y en la alusión a la homosexualidad o a la ambigüedad sexual del amado, confirmando así lo que dice Todorov con relación a los temas del otro en la literatura fantástica ${ }^{15}$.

Los versos de Twelfth Night que aparecen desde el primer capítulo dentro del fluir del pensamiento de la protagonista, anticipan los anhelos de Eleanor, promesa al lector de algo por venir. La autora destaca entre comillas el verso de Shakespeare, como respetuosa marcación del discurso ajeno que irrumpe en la mente de Eleanor. Este verso forma parte de la segunda estrofa de la canción que canta el bufón "Feste" en el Acto II, escena iii, y corresponde a la línea 48.

En la versión en español se optó por incorporar los versos traducidos sin ninguna referencia ni nota al pie. Los lectores sin duda se darán cuenta que están frente a un "discurso ajeno" y verán su relación con la situación en que surgen en el pensamiento de la protagonista. A continuación transcribimos el texto original de la canción y las dos versiones traducidas que se han obtenido en español:

\section{Original}

\section{FESTE}

(Sings)

0 mistress mine! Where are you roaming?

0 , stay and hear: your true love's coming,

\section{FESTE}

(Sings)

What is love? 'Tis not hereafter;

Present mirth hath present laughter

13. Barbara Melchiori, "Introducción" en William Shakespeare, Noche de Reyes o Como queráis - Twelfth Night or What you Will (edición bilingüe del Instituto Shakespeare dirigida por M.A. Conejero, Barcelona: Ediciones Altaya, 1994) 30-34.

14. Martínez Estrada, XXXIV.

15. Tructan Todorov (1970, The Fantastic. A Structural Appoach to a Literary Genre. Niıc va York: ('ornell University Press, 1975) 131. 
That can sing both high and low.

Trip no further pretty sweeting;

40 Journeys end in lovers meeting,

Every wise man's son doth know. (182)

Traducción (1988)

FESTE

(Canta)

Oh, amada mía, vos ¿a dónde vais?

¿No oís el canto del que tanto os ama?

¿No oís un canto, por lo bajo canto, por

[lo alto canto.

Oh, amada mía, ¿vos a dónde vais?...

Sien un breve instante, un feliz amante, todo el mundo sabe, ¿más placer os da?

\section{FESTE}

(Canta)

¿Qué es amor? Amor no es siempre.

Amor es sonrisa y gozo.

Es ahora, no es mañana:

Dadme, os pido, una razón para esperar.

Bésame amor, más de mil veces,

que soy mancebo y la hermosura se va...
What's to come is still unsure.

In delay there lies no plenty-

Then come kiss me, sweet and twenty,

Youth's a stuff will not endure. (184) 50

\section{Traducción (1870/1960)}

Bufón. (Canta) ¿Dónde vas mi bien errante, Lejos de tu fiel amante?

Ven y escucha mi canción.

No te apartes, vida mía,

Que de amor en la porfía

Triunfa el firme corazón.

Bufón. (Canta) ¿Qué es amor? No un bien

I futuro:

Lo presente está seguro,

Incierto lo porvenir.

Dame un beso, por tu vida;

Mira que la edad florida

Poco se tarda en morir. (223)

Las dos traducciones corresponden a épocas muy diferentes. La primera, junto con el texto en inglés, corresponde a la edición bilingüe del Instituto Shakespeare dirigida por Manuel A. Conejero reeditada en 1995, pero publicada por primera vez en 1988. La segunda pertenece a Jaime Clark y, según informa Bárbara Melchiori es la primera traducción al español publicada en España (1870-1876) y la 
primera en trasladar el verso de Shakespeare a nuestra lengua ${ }^{16}$ : más de un siglo ha transcurrido pues entre ambas versiones.

A pesar de sus méritos, no hemos podido escoger ninguna de las dos versiones para nuestro texto. Analicemos brevemente por qué. De la comparación de la canción en inglés y sus traducciones resulta evidente que no hay siempre una correspondencia verso por verso, aunque sí se conserva el sentido de cada estrofa. Ambas traducciones refle jan así que, en la primera, el amante alienta a su amada y le anuncia su encuentro con el verdadero amor y en la sigunda, la urge a gozar de ese amor en el presente, pues el futuro es incicrlo y la juventud efímera. Por otra parte, el desconocimiento de la meloclíi de la tonada que acompañaba a esta canción dificulta aún más salocr cual sería la traducción más apropiada con relación a la métricia, a la rima y demás aspectos del lenguaje poético. No obstante, sí sial)emos que los versos escogidos de forma aislada por Shirley Jackson recpuicren una traducción más literal, conforme con las circunstinciass en que ellos se presentan en la novela. En esta ocasión, cl verso "In clclay there lies no plenty" (22) ${ }^{17}$ se ha traducido como: "No hallarćis plenitud en la espera". Corresponde hacer dos comentarios con respecto al uso verbal, el primero con relación al paradigmal ck "vos" para un solo destinatario, es decir que usamos el verbo ('n) lis sicgunda persona del plural tal como se utilizaba a partir del siglo XIV ${ }^{18}$ y de la misma manera que lo utiliza Conejero en la traducción (juc transcribimos en primer término. El otro se refiere al uso (c) tic(mp) futuro del modo indicativo para traducir un verbo que en la versión inglesa está en presente. Esto es posible porque estamos utilizandu, lal como lo señala el Esbozo, el "futuro de probabilidad" por cl (yuc "(xpresamos suposición, conjetura (que es nuestro caso) () valcilación referidas al presente"

16. Melchiori, 61.

17. Jackson, 22.

18. Real Academia Española, Esbozo de una mu'va grumuílicu de la lengua española (Madrid: Espasa-Calpe, 1989) 339-340.

19. Real Academia Española, 471. 
Otra autora y otro texto se entrelazan con nuestra novela: la descripción del cuarto de Theodora (44), decorado en tonos de verde, guarda bastante similitud con la que hace Elizabeth Barrett Browning (1806-1861) en su poema "Room for an adolescent":

...The walls

Were green; the carpet was pure green; the straight

Small bed was curtained greenly; and the folds

Hung green about the window,... ${ }^{20}$.

En este caso, Gilbert y Gubar ven en el cuarto verde una representación del paraíso y un símbolo de la poesía ${ }^{21}$.

Jackson parece enmarcarse en una tradición que transforma la casa encantada de la leyenda en una metáfora de la mente humana, como lo veíamos cuando hablábamos de la perspectiva femenina ${ }^{22}$. Así encontramos significativas relaciones intertextuales con el siguiente poema de Emily Dickinson (1830-1886) que comienza:

One need not be a Chamber - to be Haunted-

One need not be a House-

The Brain has Corridors - surpassing Material Place__23

Poema que para Gilbert y Gubar habla del significado del Gótico, especialmente para las mujeres, género que brinda metáforas que reflejan los turbulentos estados psicológicos de disociación interior en que a menudo caían las escritoras del siglo $\mathrm{XIX}^{24}$.

20. Elizabeth Barrett Browning, "Room for an adolescent", citado por Gilbert y Gubar, 644.

21. Gibert y Gubar, 644 .

22. Dianda Martínez, 118-119.

23. Emily Dickinson, poema citado por Gilbert y Gubar, 624.

24. Gilbert y Gubar, 624 . 
Este análisis, aunque incompleto, sin embargo proporciona importantes elementos para enriquecer la lectura y por ende la traducción. Quien traduce debe limitarse a reconocer estas presencias intertextuales y a hacerlas evidentes en el texto terminal para que quien lo lea pueda encontrar los nexos, pero sin hacerlos más explícitos porque si no alteraría la magia del original: esa cierta ambigüedad que le permite hablar y sugerir muchos significados. Significados que se enriquecen aún más porque están llenos de implicaciones, de símbolos.

\section{Simbolismo en The Haunting of Hill House}

La traducción, entendida como proceso de interpretación y reconstrucción de un texto en otra lengua y en otra cultura, implica un reconocimiento de significaciones y símbolos en la cultura donde se produce el texto que no necesariamente son equiparables en la cultura terminal. En nuestro caso, el traducir un texto de la cultura estadounidense de los años 50 a la nuestra -latinoamcricana y contemporánea-, aunque ambas se nutren de raíces occidentales, requiere un análisis que siempre va a enriquecer las posibilidades de interpretación del sentido y su reelaboración en el texto terminal.

Repasemos los símbolos presentes en los capítulos traducidos y sus posibles significados en relación con la tcmática femenina que mencionan Gilbert y Gubar. Ya hemos indicado "la casa" con una presencia tan importante que adquiere dimensiones de personaje y así forma parte del título. De acuerdo a J. A. Pérér. Ri(oja, la casa es la representación freudiana típica de la personalidad humana ${ }^{25}$. "La madre", que según Jung representa al inconsciente colectivo, vimos que involucra también todo lo recibido y repctido culturalmente ${ }^{26}$. "El automóvil”, en el que Eleanor viaja y que es el arma quc escoge para suicidarse, - símbolo social de progreso, biencstar c independencia

25. J.A Pérez Rioja, Diccionario de símbolos y milos (Madrid: 'T'econos, 1994) 115.

26. Péréz. Rioja, 283. 
económica, también para la mujer-, es instrumento de muerte, escape y suicidio, al igual que lo fue para Willy Loman protagonista de Death of a Salesman (1949) de Arthur Miller, personaje con quien Eleanor comparte significativas características. "El camino", siguiendo el análisis de Malcolm Bradbury ${ }^{27}$, sin duda comparte el simbolismo de Kerouac en su narración En el camino (1957) cuando habla de un viaje liberador, lejos de la ciudad tecnificada (exterior) y otro hacia una libertad emocional (interior). "La tacita de estrellas" que con tanta ternura aparece por primera vez (21) y se repite a lo largo del texto, adquiere un claro simbolismo genital femenino ${ }^{28}$ que la psicología atribuye a toda forma cóncava, pero es una metáfora muy rica y sugiere más significados. El color azul del dormitorio de Eleanor que, según Pérez Rioja, está asociado con vivencias psíquicas, con lo espiritual, el color de la función de la mente, es un color femenino, símbolo de Venus y también "un espacio para la propia expansión"29. "Las colinas" o montañas se asocian, entre otros significados, con lugares $\operatorname{sagrados}^{30}$. "Las ventanas", según Gilbert y Gubar simbolizan mirar hacia afuera, tener proyectos, expectativas, mientras que "los espejos" implican mirar hacia adentro, una búsqueda interior y también conflictos de identidad: entre madre e hija, mujer y mujer, conflictos del $\mathrm{Yo}^{31}$. "Los árboles" y "el agua" para Jung representan lo maternal; el agua es también una de las "representaciones de la energía psíquica como fuerza pasiva femenina" y simboliza al mismo tiempo lo maternal, lo femenino y lo intrauterino ${ }^{32}$. "El bosque" sería una representación psicológica de nuestro inconsciente y "las bellas jóvenes perseguidas en el bosque”( 173-177) simbolizan nuestras malas relaciones con las figuras psíquicas interiores ${ }^{33}$. "La sangre” participa de las cualidades

27. Malcolm Bradbury, La novela norteamericana moderna (México: Fondo de Cultura Económica, 1988).

28. Pérez Rioja, 391.

29. Pérez Rioja, 88.

30. Pérez Rioja, 308.

31. Gilbert y Gubar, 37.

32. Pérez Rioja, 73-74 y 48-49.

33. Pérez Rioja, 98. 
del color rojo y se la relaciona con el sexo y emociones de amor y odio $^{34}$. Y así la lista sería demasiado larga y tampoco agotaríamos la múltiple asociación de simbolismos. En todo caso, este aspecto y lo indicado previamente en "Intertextualidades" parecen confirmar las investigaciones de Gilbert y Gubar con relación al Gótico femenino, su contenido y su forma. Para la traducción es un aspecto que no puede ser descuidado por las implicaciones culturales que podrían alterar los valores simbólicos en el texto meta.

\section{Conclusión}

El análisis de la forma en que la novela se relaciona con otrọs textos - mitológicos, tradicionales y contemporáneos a la autora-y los simbolismos presentes, junto con el de aquellos aspectos que forman la macroestructura en que se produce la novela, fue indispensable para reconocer la intención del texto y para poder interpretar de manera más acabada su sentido y así reconstruirlo en la versión en nuestra lengua. La información recogida junto con la investigación sobre la autora y su obra, el contexto histórico y literario, el género utilizado, y lo que han visto en él en el pasado y en la actualidad la crítica masculina y femenina constituyen la "basc de datos" indispensable para la comprensión del texto, es decir, para llevar a cabo una lectura activa como corresponde a un lector idóne(o, el tipo de lector que se requiere que sea el traductor literario.

Vemos que Jackson además de abordar una historia de horror nos enfrenta a los conflictos de una mujer en su búsqueda de amor. Alude a la homosexualidad y a un tema tabú: el incest() y sus consecuencias psicológicas. Pero también, con gran riqueza de recursos se refiere a muchos de los temas que preocupaban a sus contemporáneos, masculinos y femeninos: la disociación entre el individuo y la sociedad, los trastornos de la personalidad y sobre todo la condición femenina en

34. Pérez Rioja, 379. 
general y en particular en relación con la literatura. ¿Y cómo lo hace? Con una esmerada elaboración y utilizando su texto para recrear otros, reconstruyéndolos o parodiándolos. The Haunting of Hill House es una novela hecha con dedicación, construida racionalmente y también con exquisita inspiración; se merece por tanto, como toda obra literaria, una traducción elaborada después de muchas lecturas, no sólo del mismo texto, sino de tantos como sea necesario hasta que la versión traducida surja casi espontáneamente en la lengua meta. A esto por lo menos debemos aspirar quienes deseamos traducir literatura. 